\title{
La Ascendencia y el Trato Personal Como Factores Para Concretar la Procuracion de Fondos
}

\author{
Dr. Gaspar Eduardo Fuentes Garza,
}

Instituto Tecnológico y de Estudios Superiores de Monterrey, Mexico

URL:http://dx.doi.org/10.19044/esj.2020.v16n25p64

\section{Resumen}

El objetivo de este artículo es analizar el impacto que tienen dos factores importantes en el proceso de procuración de fondos. Uno de ellos es la ascendencia entre quien solicita el recurso y el posible donante y el segundo es la forma en que se establece el contacto entre ambas partes. Para llevar a cabo este estudio se documentaron los resultados de cuatro eventos de procuración de fondos realizados en distintas ciudades, pero con la misma metodología y en los cuales, participaron doscientos promotores, quienes documentaron en lo particular los resultados del proceso de solicitud de donativos con los donantes a quienes contactaron. Ambos factores impactaron de forma positiva el proceso de procuración de fondos en cada uno de los cuatro eventos. La ascendencia brinda un mayor grado de confianza a la hora de solicitar a una persona algún tipo de ayuda y la solicitud en persona de dicho apoyo también incrementa las posibilidades de que un individuo se convierta en donante.

Palabras claves: Ascendencia, filantropía, donativos 


\title{
Ascendance and Personal Treatment as Factors to Concrete the Raising of Funds
}

\author{
Dr. Gaspar Eduardo Fuentes Garza, \\ Instituto Tecnológico y de Estudios Superiores de Monterrey, Mexico
}

\begin{abstract}
The objective of this article is to analyze the impact that two important factors have on the fundraising process. One of them is the ascendancy between the person requesting the resource and the possible donor and the second is the way in which contact is established between both parties. To carry out this study, the results of four fundraising events held in different cities but with the same methodology were documented, in which 200 promoters participated, who documented the results of the donation request process with the donors who were contacted. Both factors positively impacted the fundraising process at each of the four events. Granting provides a greater degree of confidence when requesting some kind of help from a person and requesting such support in person also increases the chances of an individual becoming a donor.
\end{abstract}

Keywords: Ascendancy, philanthropy, donations

\section{Introduction}

En México, el Directorio de Donatarias Autorizadas por el Sistema de Administración Tributaria publicado en el Anexo 14 de la Resolución Miscelanea Fiscal en el Diario Oficial de la Federación, establece que en Enero de 2020, existen 14,287 instituciones donatarias (SAT, 2020).

Para estas instituciones es muy importante contar con esta categoría ya que parte de su presupuesto operativo, con el cual pueden cumplir su objeto social, proviene de donativos procedentes de empresas, personas físicas, fundaciones u otras instituciones de la sociedad civil.

El reto que tienen las organizaciones donatarias en México para obtener recursos es grande ya que la cultura filantrópica no es tan fuerte. En el informe 2018 del World Giving Index, México ocupa el lugar 114 de 144 países (CAF, 2018).

Una campaña financiera implica inversión de tiempo y recursos para las donatarias y ambos insumos son limitados. Por lo anterior, es importante que durante la campaña se consideren ciertos factores y estrategias que 
maximicen la procuración de fondos para y potencialicen la inversión realizada en favor del objetivo que se busca en la institución.

En la presente investigación se analizarán dos de estos factores, la ascendencia entre un procurador de fondos y un posible donante y la forma de realizar el contacto para hacer la solicitud.

\section{Marco Teórico}

Una donataria autorizada es una organización civil o fideicomiso, que cuenta con autorización por parte del Servicio de Administración Tributaria (SAT), para recibir donativos deducibles del Impuesto Impuesto Sobre la Renta, pueden ser Asociación Civil, Sociedad Civil ó Institución de Asistencia Privada (SAT, 2013).

Las 14,287 instituciones donatarias autorizadas en México son agrupadas por el SAT en 12 categorías las cuales son: Asistenciales, educativas, investigación científica, culturales, becantes, ecológicas, para la reproducción de especies en protección y en peligro de extinción, apoyo económicode otras donatarias autorizadas, obras ó servicios públicos, propietarias de bibliotecas privadas con acceso al público en general, propietarias de museos privados con acceso al público en general y desarrollo social (SHCP, 2018).

Algunas de las ventajas que tiene para una institución el ser donataria están: tributar para efectos fiscales como persona moral con fines no lucrativos, puede recibir donativos sin límite, ya sea en efectivo o en especie, de residentes en el país o en el extranjero, debiendo expedir los comprobantes respectivos y de manera general no es contribuyente del Impuesto Sobre la Renta (SAT, 2020).

Las personas físicas y personas morales pueden deducir los donativos no onerosos ni remunerativos que den a las personas morales autorizadas para recibir donativos deducibles, siempre y cuando, el monto total de dichos donativos no exceda $7 \%$ de los ingresos acumulables que sirvan de base para calcular el impuesto sobre la renta (SAT, 2020).

Las donatarias con programas asistenciales, de educación, investigación científica, culturales y que brindan apoyo a otras donatarias, son las que más recursos en efectivo reciben en México (SHCP, 2018).

Los donativos que reciben estas instituciones vienen de donantes institucionales o de sus mismos fundadores y con esto pueden llevar a cabo su operación. Otras, además del tipo de recursos mencionados anteriormente, requieren llevar a cabo actividades que permitan obtener fondos de personas físicas y morales y así poder cumplir sus objetivos y proyectos.

La procuración de fondos es el esfuerzo de un grupo de personas comprometidas con una causa por la cual trabajan en forma organizada y 
eficiente para alcanzar una meta económica definida, en un periodo determinado de tiempo (CIJ, 2018).

Hay varias maneras de implementar modelos de procuración de fondos Albergue Infantil Los Pinos (2018):

1. Correo directo

2. Recaudación por teléfono

3. Petición cara a cara

4. Avisos en prensa y radio

5. Recolecta en la calle

6. Recolecta en las iglesias

7. Escuelas

8. Tiendas de beneficencia

9. Ventas por correo

10. Donantes que aportan pequeñas cantidades de dinero.

11. Sorteos.

De estos modelos, una opción es la petición directa a través de campañas anuales o principales donde se buscan donantes con una alta capacidad económica que les permite hacer donativos de monto elevado. Esto ayuda a las organizaciones a llegar a los montos de meta requeridos con pocos donantes. Y la otra forma es a través de la búsqueda de donativos de monto pequeño, el cual se puede cobrar de forma mensual recurrente por al menos un año y con una base amplia de donantes que en el largo plazo se convierte en otra fuente importante de ingresos. (CIJ, 2018).

Para cualquiera de estos dos modelos de procuración se requiere hacer contacto entre un solicitante y un posible donante y que entre ambos exista un compromiso el cual, además de permitir el resultado positivo de la petición, se deberá de seguir fortaleciendo para darle al donante la seguridad de que la causa que está apoyando se está cumpliendo. (GG+A,2020).

Como se describe anteriormente el contacto entre dos personas requiere que existan una buena ascendencia, es decir, el predominio moral o influencia que existe entre ellos (RAE, 2020). Y también el medio de contacto para establecer la comunicación entre ambos.

\section{Metodología}

Esta investigación busca determinar el impacto que tienen en la gestión de donativos, la ascendencia entre el solicitante y el donante así como la forma de contacto en la que se hizo dicha solicitud de donativo.

El proceso de evaluación se realizó con una metodología descriptiva que explica en detalle como se da el contexto de la situación. 
Se empleó la investigación cuantitativa para medir el resultado de utilizar los dos factores de ascendencia y contacto en la procuración de fondos. Para lograr lo anterior, se documentaron las solicitudes que 200 promotores en cada una de las 4 ciudades donde se llevaron a cabo procesos de procuración de fondos.

\section{Detalle de la Implementación}

Para este estudio se dio seguimiento a cuatro eventos de procuración de fondos llevados a cabo en cuatro ciudades del norte de México.

Dichos eventos se desarrollaron bajo un mismo modelo de procuración el cual se explica a continuación:

1. Se presenta a un grupo de 20 personas un proyecto el cual requiere recursos económicos para poder llevarse a cabo.

2. A dicho grupo se les invita a participar en una campaña de cinco semanas en la cual ellos serán líderes de equipos los cuales estarán conformados por empresarios, altos directivos, líderes de opinión, académicos y personas en general afines a la causa.

3. Estos 20 líderes invitan al menos a diez amigos para formar un equipo de campaña.

4. En total se arma un grupo de al menos 200 promotores que buscarán recursos a favor de la causa elegida.

5. Cada uno de los promotres del equipo se compromete a conseguir un monto mínimo de donativos de $600 \mathrm{MXN}$ por semana, los cuales se cobrarán mensualmente.

6. Al final de las 5 semanas, cada miembro del equipo habrá procurado al menos donativos por 3,000 MXN mensuales que se cobrarán de forma recurrente cada mes.

7. 200 promotores que logran donativos de 3,000 MXN mensuales generan cada mes 600,000 MXN los cuales en un año sumarán 7,200,000 MXN.

8. Los donativos se captan a través de una plataforma virtual que registra la información del donante y hace los cargos mensuales así como también el reporteo de información para temas de transparencia en el uso de los recursos.

9. Además de los donativos mensuales recurrentes, los promotores pueden hacer solicitudes de donativos únicos de mayor monto en caso que el perfil del donante sea adecuado.

Los 200 promotores de cada ciudad compartieron su numero de gestiones exitosas tanto con el factor de ascendencia así como por el tipo de contacto realizado. 
Para que cada promotor pudiera llegar a su monto mínimo donativos esperados de 3,000 MXN mensuales recurrentes, fue necesario que hicieran contacto con varios posibles donantes en búsqueda de una respuesta positiva.

La media del número total de solicitudes de donativos realizada por los promotores durante las 5 semanas de la campaña fue de 18 gestiones.

Se llevó un registro de las gestiones por promotor para con dicha información, poder hacer el análisis correspondiente. En dicho reporte el promotor registraba si había ascendencia con la persona a la que se hizo la petición de donativo y para quienes aceptaron donar, también se registró la forma de contacto que se tuvo con ellos.

\begin{tabular}{|c|c|c|c|c|}
\hline Promotor: & \multicolumn{3}{|l|}{} \\
\hline Donante & $\begin{array}{c}\text { Es conocido (Con } \\
\text { Ascendencia) }\end{array}$ & $\begin{array}{c}\text { No es conocido } \\
\text { (Sin Ascendencia) }\end{array}$ & Sí donó & No donó \\
\hline Donante 1 & & & & \\
\hline Donante 2 & & & & \\
\hline$\ldots$ & & & & \\
\hline
\end{tabular}

Fuente: elaboración propia

La información recabada se concentró para poder analizarla.

\section{Resultados}

En las siguientes tablas se muestran los registros de los resultados obtenidos durante el proceso de procuración de fondos en las cuatro ciudades donde se llevaron a cabo.

Tabla 1. Resultados positivos obtenidos por un promotor al solicitar un donativo a un contacto con el que se cuenta con y sin el factor de ascendencia:

\begin{tabular}{|l|c|c|c|c|}
\hline Factor / Ciudad & Ciudad 1 & Ciudad 2 & Ciudad 3 & Ciudad 4 \\
\hline Con Ascendencia & $72 \%$ & $68 \%$ & $74 \%$ & $73 \%$ \\
\hline Sin Ascendencia & $5 \%$ & $6 \%$ & $5 \%$ & $7 \%$ \\
\hline
\end{tabular}

Fuente: elaboración propia.

Tabla 2. Distribución de resultados positivos al solicitar un donativo en base al tipo de contacto hecho por el promotor.

\begin{tabular}{|l|c|c|c|c|}
\hline \multicolumn{1}{|c|}{$\begin{array}{c}\text { Contacto } \\
\text { /Ciudad }\end{array}$} & Ciudad 1 & Ciudad 2 & Ciudad 3 & Ciudad 4 \\
\hline Personal & $84 \%$ & $87 \%$ & $85 \%$ & $83 \%$ \\
\hline $\begin{array}{l}\text { Teléfono } \\
\text { /Video }\end{array}$ & $9 \%$ & $7 \%$ & $9 \%$ & $10 \%$ \\
\hline $\begin{array}{l}\text { Correo } \\
\text { electrónico }\end{array}$ & $5 \%$ & $3 \%$ & $4 \%$ & $2 \%$ \\
\hline Chats & $2 \%$ & $3 \%$ & $2 \%$ & $5 \%$ \\
\hline
\end{tabular}

Fuente: elaboración propia. 
Los resultados obtenidos respecto al impacto de la aplicación de los dos factores para aplicar en una campaña financiera fueron:

- La ascendencia entre el promotor y donante nos da un 70\% de probabilidad de que se tenga una respuesta positiva ante una petición de apoyo para una causa determinada.

- Cuando no hay ascendencia entre el promotor y donante la probabilidad de que haya rechazo en una petición de donativo, supera el $90 \%$.

- En los casos que la respuesta del donante fue positiva la forma de contacto más exitosa fue la presencial con más de $80 \%$ de éxito, un segundo lugar fue la llamada telefónica la cual estuvo cerca del $10 \%$.

- Los correos electrónicos y los chats como medio de contacto para solicitar un donativo en conjunto suman un $7 \%$ de probabilidad de éxito.

\section{Conclusiones}

Partiendo de los dos factores analizados en esta investigación los cuales fueron: el nivel de ascendencia entre el promotor y el donante así como el medio de contacto. Podemos concluir que para tener un mayor éxito en la procuración de fondos, es importante lo considerar lo siguiente:

1. Las solicitudes de donativos a posibles donantes, deben hacerse a través de personas con la suficiente ascendencia para que la probabilidad de una respuesta negativa sea menor.

2. La forma de contacto entre el posible donante y un promotor, que brinda una mayor probabilidad de generar una respuesta positiva es mediante el trato en persona.

3. Una llamada entre amigos con suficiente ascendencia también puede dar un nivel de resultados positivos.

4. Los chats individuales ó grupales, así como los correos electrónicos no son el medio de contacto más recomendable para hacer una petición de donativos tanto para montos elevados, así como para montos de menor cantidad.

En futuras investigaciones se podrían analizar otros factores que puedan potencializar el resultado positivo a la hora de solicitar un donativo.

El resultado obtenido con este trabajo permitirá a quienes realizan tareas de procuración conocer al menos el resultado de contemplar estos dos factores.

En México, aún falta mucho para desarrollar una mayor cultura filantrópica (CAF, 2018), los vínculos entre las personas, así como el trato directo se convierten en dos criterios claves para convencer a un público sobre 
la importancia de ayudar sumándose a causas filantrópicas que ayuden a transformar la realidad de nuestra sociedad.

El aplicar estas estrategias en los procesos de procuración de fondos podrán favorecer el resultado de esperado.

\section{References:}

1. SHCP. (2018). Reporte de Donatarias Autorizadas. Recuperado de https://www.gob.mx/cms/uploads/attachment/file/394640/Reporte_de _Donatarias_Autorizadas_2018.pdf

2. CAF. (2018). Word Giving Index. Recuperdo de https://www.cafonline.org/docs/default-source/about-uspublications/caf_wgi2018_report_webnopw_2379a_261018.pdf?sfvr sn=c28e9140_4

3. SAT. (2013). Donatarias autorizadas. Recuperado de https://www.senado.gob.mx/comisiones/relext_ongs/mesas/docs/mes a2_SAT.pdf

4. SAT. (2020a). Directorio de Donatarias Autorizadas. Recuperado de https://www.sat.gob.mx/consultas/27717/conoce-el-directorio-dedonatarias-autorizadas

5. SAT. (2020b). Conoce las ventajas de ser donataria autorizada. Recuperado de https://www.sat.gob.mx/consulta/70077/conoce-lasventajas-de-ser-donataria-autorizada

6. Centro de Integración Juvenil (2018). Procuración de Fondos : Saber pedir para obtener. Recuperado de http://www.cij.gob.mx/patronatosCIJ/pdf/RecaudacionFondos.pdf

7. Real Academia Española (2020). Recuperado de https://dle.rae.es/ascendencia

8. Los Pinos (2020). Conceptos básicos y procuración Fondos. Recuperado de:

https://www.lospinos.org/wp-content/uploads/2016/10/manual-deprocuracion-de-fondos.pdf

9. GG+A. (2020) Grenzebach, Glier and Associates. The basic rules of engagement haven't changed, but the pandemic has created new opportunities and challenges. Recuperado de:

https://www.grenzebachglier.com/2020/08/06/the-basic-rules-ofengagement-havent-changed-but-the-pandemic-has-created-newopportunities-and-challenges/ 